\title{
Calculation of the resource use limiting factors in the management digital transformation context
}

\author{
Olga Milekhina*, and Irina Aslanova \\ Novosibirsk State Technical University, 630087 Novosibirsk, Russia
}

\begin{abstract}
On the basis of the equation of entropy-information balance, which can describe the macromodel of control of equipment operation, consumption of materials, energy carriers, the limiting values of the coefficients of their use and losses are obtained. It turned out that the ratio of the latter satisfies with high accuracy the proportions of the golden section. This quantitative regularity, which is of a systemic nature, should be taken into account as a standard in the feasibility study of projects of information systems for production management, which has a probabilistic nature.
\end{abstract}

\section{Introduction}

Traditional analytics in the process of making management decisions relied on a cascade process of collecting, processing, storing data. It was supposed to perform iterative actions to form a hypothesis and test it against the available data. Their lack was compensated by the practical experience of the decision-maker, adoption of the best practices, and the results of consulting with experts. Next, a gradual analysis of small data packets was carried out, reduced during the sorting process before processing. However, there was no significant improvement in the quality of management decisions made, since the use of information systems in organizations was rarely accompanied by a noticeable increase in the range of processed data [1,2], it required an increase in subjectivity in business processes [3], their system parameterization [4], revision of the views of managers on the area of benefits from analytical information systems $[5,6]$.

Digitalization symbolized the process of completing the transition from disparate information systems to digital business ecosystems [7], created fundamentally new business models of SaaS services (English System as a Service, SaaS) and DaaS (English Desktop as a Service, DaaS), radically transformed analytical activity in the process of making management decisions (Fig. 1).

\footnotetext{
${ }^{*}$ Corresponding author: olga.milekhina@gmail.com
} 


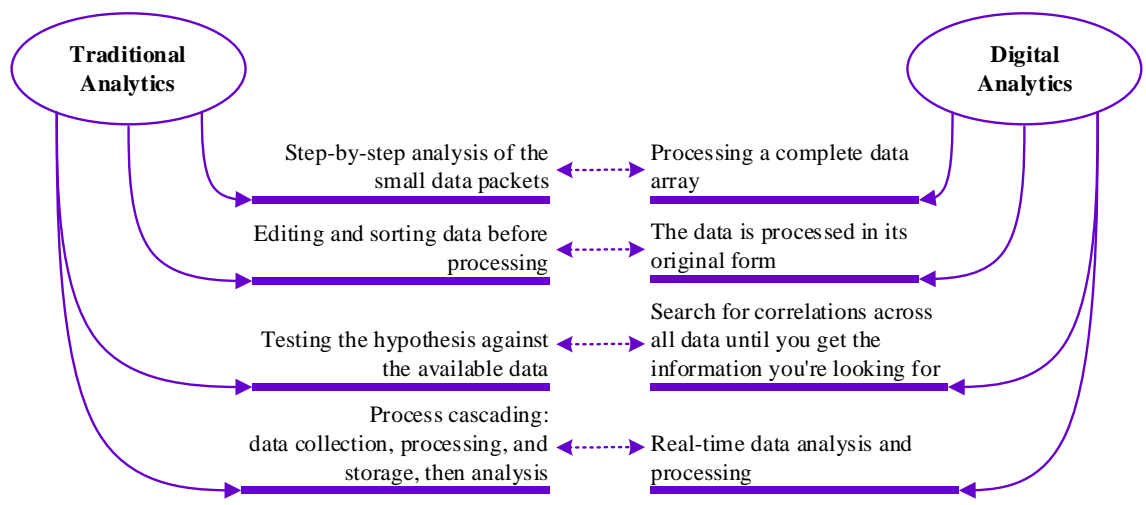

Fig. 1. Components of analytical activities based on traditional and digital analytics.

Digital technologies provided the possibility of data-based management and allowed analysts to process the entire data array at once in their original form, look for correlations across all data until the desired information is obtained, analyze and form a management decision in real time (Real Time Enterprise, RTE) [8-14].

The creation of digital business ecosystems allows a systematic approach to solving the problem of maximizing the use of existing reserves by improving the quality of business process management in organizations. Of particular interest is the question of the maximum possible use of available reserves by improving the quality of management of industrial facilities. Despite the external differences, they have a common and essential feature: this is an incompletely deterministic, largely probabilistic nature of their functioning.

\section{Methods}

Theoretical prerequisites of the solution to this problem are offered in the research of academician V.A. Trapeznikov [15]. On the basis of a macro-approach to the study of complex systems, using the concepts of entropy and the amount of information, he established a number of fundamental laws of information systems. Academician V.A. Trapeznikov claimed that the main objective of manufacturing execution systems is the reduction of its inherent partial disorder, the measure of which is entropy. However, as has been shown, achieving an arbitrarily small value of this value is not economically feasible. This approach was further developed, in particular, in $[1,16]$. Below, using the methodology used in them, the limiting (optimum by the cost/effect criterion) values of the resource utilization factors are found.

\section{Results and discussion}

Let us designate $\mathrm{R}$ the total cost of any resource consumed by an object during the planning period of time, and $E$ as the cost of its useful part. Then the relations $\frac{E}{R}=p$ and $\frac{R-E}{R}=q=$ $1-p$ (i.e. the coefficients of use and losses) can be considered as probabilities of corresponding "progress" and "failures". Since these events form a full group, their entropy will have an appearance $[16,17]$ :

$$
H(p, q)=-(p \log p+q \log q)=-[p \log p+(1-p) \log (1-p)]
$$

where the basis of logarithms is equal to two. 
This function, according to its main property, reaches the maximum value at $p=q=$ 0,5 and makes $H(0.5,0.5)=-(0.5 \log 0.5+0.5 \log 0.5)=1$ that means a full disorder of a system. The transfer of the latter from this state to an acceptable value $p$, i.e. the implementation of the act of management, requires the amount of information $I$ equal to the difference of entropies:

$$
I=H(0.5,0.5)-H(p)=1+p \log p+(1-p) \log (1-p)
$$

Formula (2) defines the amount of information per one binary sign of its representation. If during the planning period the information system received $\mathrm{n}$ signs, then at the cost of a unit of information of $r$ the expense for management process will be nrI. Having designated $\mathrm{nr}=\mathrm{Q}$, the value $\mathrm{E}$, we will present size in the form $\mathrm{E}=\mathrm{Rp}-\mathrm{QI}$, wherein the costs of management are classified as losses. Considering (2), the expression for E will be

$$
E=R p-Q[1+p \log p+(1-p) \log (1-p)]
$$

From this dependence (we will call it the equation entropy - information balance) it follows that the use of information for management will change (increase) the probability $p$. However, the expenses $Q$ then will increase as well, thus at some value $p$ the value $E$ can become negative. Let us find its maximum. Solving the equation $\frac{\mathrm{dE}}{\mathrm{dp}}=0$, we receive:

$$
p_{\max }=\frac{2^{\frac{R}{Q}}}{1+2^{\frac{R}{Q}}}=\frac{1}{1+2^{-\frac{R}{Q}}},
$$

and, respectively,

$$
q_{\min }=1-p_{\max }=\frac{1}{1+2^{\frac{R}{Q}}}
$$

Since the value $\frac{d^{2} E}{d^{2}}<0$, at $p_{\max }$ the value $E$, indeed, reaches a maximum. Let us employ the designation $\frac{R}{Q}=a$ and we will make substitution (4) in (2) and (3):

$$
\begin{aligned}
& I_{\max }=1-\frac{\log \left(1+2^{\mathrm{a}}\right)}{1+2^{\mathrm{a}}}-\frac{\log \left(1+2^{-\mathrm{a}}\right)}{1+2^{-\mathrm{a}}} \\
& \mathrm{E}_{\max }=\frac{\mathrm{R}}{1+2^{-\mathrm{a}}}-\mathrm{Q}\left[1-\frac{\log \left(1+2^{\mathrm{a}}\right)}{1+2^{\mathrm{a}}}-\frac{\log \left(1+2^{-\mathrm{a}}\right)}{1+2^{-\mathrm{a}}}\right] .
\end{aligned}
$$

Having divided both parts (7) by $\mathrm{R}$, for the maximum value of efficiency $\mathrm{K}_{\max }$ we will receive:

$$
K_{\max }=\frac{E_{\max }}{R}=\frac{1}{1+2^{-a}}-\frac{1}{a}\left[1-\frac{\log \left(1+2^{a}\right)}{1+2^{a}}-\frac{\log \left(1+2^{-a}\right)}{1+2^{-a}}\right] .
$$

This size critically depends on parameter a, i.e. on the relation of total cost of the used resource to full costs of information processing in an information system. Therefore, to obtain the numerical results of the problem under consideration, it is necessary to determine the set of its admissible values. Since the entropy maximum per one sign is $H(p) \leq 1$, the corresponding amount of information will be $\mathrm{I} \leq 1$ as well. Having put probability size in square brackets (7) to equal unit, we will receive the expression $E_{\max }=\frac{R}{1+2^{-a}}-Q$ corresponding to costs of processing of the most available amount of information. A necessary condition for its correctness must be the fulfillment of the inequality $\frac{R}{1+2^{-a}}-Q>$ 0 , which, considering the designation $\mathrm{a}=\frac{\mathrm{R}}{\mathrm{Q}}$, may be written as: 


$$
\frac{1}{1+2^{-a}}>\frac{1}{a}
$$

The solution of (9) isa $\geq 1,4$ (that can be verified directly).

Further, pursuant to the criterion of maximizing $\mathrm{K}$, requiring the processing costs for $\mathrm{I}_{\text {max }}$ units of information to be lesser than the minimum losses $R q_{\min }$, i.e. $I_{\max } Q<R q_{\min }$, or $\frac{1}{\mathrm{a}} \mathrm{I}_{\max }<\mathrm{q}_{\min }$.

This requirement, considering (5) and (6), we will present in the form:

$$
\frac{1}{\mathrm{a}}\left[1-\frac{\log \left(1+2^{\mathrm{a}}\right)}{1+2^{\mathrm{a}}}-\frac{\log \left(1+2^{-\mathrm{a}}\right)}{1+2^{-\mathrm{a}}}\right]<\frac{1}{1+2^{-\mathrm{a}}}
$$

The latter inequality holds for a $\leq 2,4$ (which may be verified directly as well). It means that the information value of increase $\mathrm{K}$ is exhausted.

Thus, the joint solution of a system of inequalities (9) and (10) is the interval 1,4 $\leq \mathrm{a} \leq$ 2 ,4. Substitution of its boundary values in (8) gives the range of extreme values of efficiency of resources: $0,62 \leq \mathrm{K}_{\max } \leq 0,68$. Herewith, the relation of the minimum value 0,62 to the corresponding size of coefficient of losses 0,38 with a relative error less than a percent satisfies proportions of "golden ratio" 1,62 [18].

The results obtained above allow, at least qualitatively, to evaluate the efficiency of projects of information systems for production management of the above type.

Let us give an example. If we accept $\mathrm{K}=0,5$ as the initial value and $\min \mathrm{K}_{\max }=0,62$ as the final value, then absolute increase in a share of useful use of a resource will be $\Delta \mathrm{R}=$ $\min K_{\max }-0,5 R=0,12 R$. Costs of creation of the new version of an information system will make $Q=\frac{R}{a}=\frac{R}{1,4}$, and efficiency ratio of capital investments will be $\frac{R}{Q}=0,12 * 1,4=0,168$ that corresponds to the term of their payback of $\frac{1}{0,168} \approx 6$ years.

Similar calculation for $\mathrm{K}_{\max }=0,68$ and $\mathrm{a}=2,4$ gives $\frac{\Delta \mathrm{R}}{\mathrm{Q}}=43,2 \%$ and a payback period of 2,3 years. Cases when starting values $\mathrm{K}$ are below $50 \%$ are of special interest.

\section{Conclusion}

1. The basis of the macromodel of production management, which has a probabilistic nature, is the entropy-information balance equation (3). It establishes the dependence between such indicators as probability of a condition of the production, amount of information necessary for its purposeful change and size K (or other indicator of efficiency). The "golden section", found in the ratio of the marginal loss coefficients, testifies to the systemic nature of this dependence and its adequacy to reality. The numerical values of these coefficients should be considered as normative in the feasibility study of the projects of the corresponding digitalization projects.

2. The accepted criterion of efficiency of use of resources $K$ as noted in Item 3., is violated at $\mathrm{a}>2,4$. It means that excess $\mathrm{K}_{\max }=0,68$ due to further improvement of quality of management (i.e. use of information resource) is already impossible. However, this statement does not exclude improvement of the use of a resource in any different way. One of the many examples of this kind is provided by the electric power industry. The efficiency of powerful heat turbines at large power plants is much lower than $\mathrm{K}_{\max }$, however, the huge amount of heat generated by them during the work is wasted for cooling. It is used for heating of water in heating systems for domestic needs. 
Therefore, the deployment of digital business ecosystems, combined with the integrated use of consumed resources, allows to radically improve the overall economic efficiency of industrial facilities of the type under consideration.

\section{References}

1. G.I. Kaigorodtsev, J. Appl. Inf. 10, 5 (2015)

2. O.P. Kultygin, J. Appl. Inf. 15, 105 (2020)

3. V.G. Chebotarev, A.I. Gromov, J. B. Inf. 23, 3 (2013)

4. R.B. Vasiliev, G.A. Levochkina, J. B. Inf. 28, 15 (2014)

5. E.S. Seredenko, J. B. Inf. 13, 10 (2013)

6. O.V. Milekhina, I.B. Adova, St. Pb. St. Pol. Uni. J. Econ. 256, 97 (2016)

7. O.V. Trofimov, V.Ya. Zakharov, V.G. Frolov, Act.N.Nov.St.Uni.Ser.: Soc.Sc. 56, 43 (2019)

8. H. Plattner, A. Zeier, In-memory data management: An inflection point for enterprise applications (Springer2011)

9. P.O. Scobelev, Cons. Ont. J. 1, 6 (2012)

10. D. Kyriazis, T. Varvarigou, K. Konstanteli, Achieving real-time in distributed computing: From grids to clouds. IGI Global (2011)

11. R. Mall, Real-time systems: Theory and practice. IGI Global (2006)

12. P.A. Laplante, S.J. Ovaska, Real-time systems design and analysis: Tools for the practitioner (John Wiley \& Sons, 2011)

13. R. Oshana, Chapter 8: DSP in embedded systems. Embedded software (Elsevier, 2008)

14. R. Gimranov, Customer report: Surgutneftegas deploys SAP HANA to increase the energy efficiency of thousands of operating facilities in real time (SAP Press, 2014)

15. V. A. Trapeznikov, J. Autom. R. C. 1, 5 (1966)

16. M. L. Seleznev, Information and computing systems and their efficiency. Sov. Radio. (1986)

17. A.M. Yaglom, I. M. Yaglom, Probability and information (Nauka, 1973)

18. F. Korbalan, The golden ratio. Mathematical language of beauty (De Agostini, 2014) 